\title{
BMJ Open A systematic review of interventions by healthcare professionals to improve management of non-communicable diseases and communicable diseases requiring long-term care in adults who are homeless
}

\author{
Peter Hanlon, ${ }^{1}$ Lynsey Yeoman, ${ }^{1}$ Lauren Gibson, ${ }^{2}$ Regina Esiovwa, ${ }^{2}$ \\ Andrea E Williamson, ${ }^{3}$ Frances S Mair, ${ }^{1}$ Richard Lowrie ${ }^{2}$
}

To cite: Hanlon P, Yeoman L, Gibson L, et al. A systematic review of interventions by healthcare professionals to improve management of non-communicable diseases and communicable diseases requiring longterm care in adults who are homeless. BMJ Open 2018;8:e020161. doi:10.1136/ bmjopen-2017-020161

- Prepublication history and additional material for this paper are available online. To view these files, please visit the journal online (http://dx.doi. org/10.1136/bmjopen-2017020161).

Received 17 October 2017 Revised 1 February 2018 Accepted 19 February 2018

\section{Check for updates}

${ }^{1}$ General Practice and Primary Care, Institute of Health and Wellbeing, University of Glasgow, Glasgow, UK ${ }^{2}$ Pharmacy and Prescribing Support Unit, NHS Greater Glasgow and Clyde, West Glasgow Ambulatory Care Unit, Glasgow, UK

${ }^{3}$ General Practice and Primary Care, School of Medicine,

Dentistry and Nursing, University of Glasgow, Glasgow, UK

Correspondence to Dr Richard Lowrie; Richard.Lowrie@ggc.scot. nhs.uk

\section{ABSTRACT}

Objective Identify, describe and appraise trials of interventions delivered by healthcare professionals to manage non-communicable diseases (NCDs) and communicable diseases that require long-term care or treatment (LT-CDs), excluding mental health and substance use disorders, in homeless adults.

Design Systematic review of randomised controlled trials (RCTs), non-RCTs and controlled before-after studies. Interventions characterised using Effective Practice and Organisation of Care (EPOC) taxonomy. Quality assessed using EPOC risk of bias criteria.

Data sources Database searches (MEDLINE, Embase, PsycINF0, Scopus, Cumulative Index to Nursing and Allied Health Literature (CINAHL), Applied Social Sciences Index and Abstracts (ASSIA) and Cochrane Central Register of Controlled Trials), hand searching reference lists, citation searches, grey literature and contact with study authors. Setting Community.

Participants Adults ( $\geq 18$ years) fulfilling European Typology of Homelessness criteria.

Intervention Delivered by healthcare professionals managing NCD and LT-CDs.

Outcomes Primary outcome: unscheduled healthcare utilisation. Secondary outcomes: mortality, biological markers of disease control, adherence to treatment, engagement in care, patient satisfaction, knowledge, selfefficacy, quality of life and cost-effectiveness.

Results 11 studies were included (8 RCTs, 2 quasiexperimental and 1 feasibility) involving 9-520 participants (67\%-94\% male, median age 37-49 years). Ten from USA and one from UK. Studies included various NCDs $(\mathrm{n}=3)$; or focused on latent tuberculosis $(\mathrm{n}=4)$; HIV $(n=2)$; hepatitis $C(n=1)$ or type 2 diabetes $(n=1)$. All interventions were complex with multiple components. Four described theories underpinning intervention. Three assessed unscheduled healthcare utilisation: none showed consistent reduction in hospitalisation or emergency department attendance. Six assessed adherence to specific treatments, of which four showed improved adherence to latent tuberculosis therapy. Three
Strengths and limitations of the study

This is the first systematic review to explicitly focus on non-communicable disease (NCD) and communicable disease that requires long-term care or treatment (LT-CD) management for adults who are homeless.

- A comprehensive search strategy was supplemented with hand searching, grey literature searches and contact with study authors.

- Interventions are described using the Effective Practice and Organisation of Care taxonomy.

- Significant heterogeneity precluded meta-analysis, so a narrative synthesis is presented along with a harvest plot summarising study findings.

- Evidence available is mostly limited to the USA, with one study from the UK.

concerned education case management, all of which improved disease-specific knowledge. No improvements in biological markers of disease (two studies) and none assessed mortality.

Conclusions Evidence for management of NCD and LT-CDs in homeless adults is sparse. Educational casemanagement interventions may improve knowledge and medication adherence. Large trials of theory-based interventions are needed, assessing healthcare utilisation and outcomes as well as assessment of biological outcomes and cost-effectiveness.

\section{INTRODUCTION}

The prevalence of homelessness is increasing across high-income countries. ${ }^{1}$ The experience of homelessness is associated with increased morbidity and mortality. ${ }^{2-4}$ Social exclusion and socioeconomic deprivation, ${ }^{56}$ adversity over the life course, ${ }^{7}$ and environmental and behavioural risk factors ${ }^{8}$ typical of 
homelessness contribute to an increased prevalence of a range of health problems compared with the rest of the population. ${ }^{1}$ This review focuses on both non-communicable diseases (NCDs) and communicable diseases that require long-term care or treatment (LT-CDs), excluding mental health and substance use disorders. We take this focus because compared with interventions for mental health disorders or substance use disorders, the management of NCD and LT-CDs in the context of homelessness has not been synthesised in the systematic review literature. ${ }^{9}$ Such conditions disproportionately affect people who are homeless (eg, tuberculosis (TB) rates 20 times higher than general population, generally poorer control of diabetes and hypertension and higher cardiovascular mortality). ${ }^{1}$ Innovative models of care and expanded roles of healthcare professionals offer potential strategies to target NCDs and LT-CDs.

Outcomes of both NCDs and LT-CDs are poorer among people who are homeless. ${ }^{10} 11$ Engagement with scheduled appointments, preventative health services and adherence to treatment are typically lower. ${ }^{12-15}$ Barriers to access, conflicting priorities, physical and mental multimorbidity are thought to contribute to poorly coordinated use of healthcare services. ${ }^{15}$ Consequently, there is a need for tailored services. ${ }^{15-17}$ Healthcare delivery models for people experiencing homelessness include specialised or generalist primary care services ${ }^{18}$; and integrated housing and health interventions. There is insufficient evidence of reach and effectiveness to favour one model over another. ${ }^{19}$ The expanding role of various healthcare professionals, for example, registered nurses and pharmacists, targeting NCD/LT-CDs, ${ }^{20}$ offers a complementary model of healthcare for people who are homeless. Sharing clinical roles may be welcome given the increasing evidence of multimorbidity and polypharmacy. ${ }^{21}$

Controlled evaluations of models of healthcare for people who are homeless are relatively few and optimal delivery varies between different health and social care systems. ${ }^{17}$ There have been calls to evaluate more interventions to improve the health of people who are homeless, ${ }^{22}$ including long-term prospective studies with economic analyses.

Previous systematic reviews have identified the potential benefit of tailored interventions for addressing mental health disorders and at-risk substance use. ${ }^{23}{ }^{24}$ These have shown potential for monetary incentives to improve adherence for people who are homeless with latent TB, ${ }^{23}$ and that provision of housing improved health outcomes in HIV. ${ }^{24}$ However, to the authors' knowledge, no previous systematic reviews have specifically focused on the potential impact of healthcare professional or other intervention on NCDs and LT-CDs for adults experiencing homelessness.

\section{Aims}

This review aims to systematically identify, describe and appraise trials of interventions focusing on the management of NCD and LT-CDs, delivered by healthcare professionals for adults who are homeless. It addresses the following two research questions:

1. What are the key components of interventions delivered by healthcare professionals aimed at improving management of NCD and LT-CDs including theoretical underpinnings?

2. What impact has been demonstrated by trials of interventions delivered by healthcare professionals aimed at improving management of NCD and LT-CDs?

\section{METHODS}

This systematic review followed a prespecified protocol $^{25}$ (registered with International Prospective Register of Systematic Reviews, ID: CRD42016046183, available at http://www.crd.york.ac.uk/PROSPERO/ display_record.asp?ID=CRD42016046183) and is described according to the Preferred Reporting Items for Systematic Reviews and Meta-Analyses (PRISMA) statement. ${ }^{26}$

\section{Eligibility criteria}

Eligibility criteria and search process are described in detail in our published protocol paper, ${ }^{25}$ and are outlined briefly below. Full details are given in online supplementary file 1 . Homelessness was defined according to the European Typology of Homelessness (ETHOS) criteria. ${ }^{27}$ Eligible studies included adult participants who met the ETHOS-defined homelessness criteria with one or more NCDs or LT-CDs or those concerning management of these conditions as part of a broader intervention (eg, access to primary care). We considered any change to the organisation or delivery of care to be an intervention. Delivery by a healthcare professional was required, defined as a person with professional training or registration to provide healthcare. Peer-health advisors (lacking professional training) and social workers (lacking health-specific training) were not considered healthcare professionals, however, interventions involving a wider range of roles were eligible for inclusion if a healthcare professional was involved in delivery as part of a wider team.

We considered a range of prespecified outcomes. Studies including any of our primary or secondary outcomes were eligible for inclusion. Unscheduled healthcare utilisation was our primary outcome. Secondary outcomes included physical measures of disease control, quality of life, behavioural outcomes, emotional well-being, satisfaction with care and cost-effectiveness. These are fully detailed in online supplementary file 1 .

\section{Literature search}

MEDLINE, Embase, Scopus, PsycINFO, CINAHL, ASSIA and Cochrane Central Register of Controlled Trials were searched from 1966 (or inception) to October 2016. The search was updated in November 2017. Our search strategy was 'homelessness' and 'NCD/LT-CDs or healthcare delivery terms' and 'trial or evaluation 
terms'. The full search terms for MEDLINE are shown in online supplementary file 1 and were adapted for other databases. Database searches were supplemented by hand searching of reference lists of all eligible studies, hand searching the Journal of Health Care for the Poor and Underserved, and forward citation searches of included studies using Web of Science. A number of 'grey Literature' sources were also searched (online supplementary file 1). Grey literature and relevant conference abstracts were used to identify recently published studies.

Two reviewers (PH plus LY, RL or RE), using DistillerSR software, independently screened titles and abstracts of all records identified. Full texts of all potentially eligible studies were obtained and assessed independently by two reviewers (PH, LY or RE) against the eligibility criteria. At all levels disagreements were resolved by discussion, involving a third reviewer (RL or LY) when consensus could not be reached. Where studies included homeless participants but analysis of these participants was not presented separately, we contacted the study authors to request these data. Studies were excluded if these were not available. Using a standardised data extraction form, two reviewers (PH plus LY or LG) independently extracted data from each study eligible for inclusion. The components of each intervention were described according to the Cochrane Effective Practice and Organisation of Care (EPOC) taxonomy. ${ }^{28}$ Two reviewers independently assessed each study according to the criteria outlined in the Cochrane EPOC guidelines for assessing risk of bias (ROB) in randomised controlled trials (RCTs), non-RCTs and controlled before-after (CBA) studies. $^{28}$ After grading each study, a judgement of the overall ROB was made for each outcome, taking into account the relative importance of potential sources of bias to the outcome in question.

\section{Synthesis}

We assessed the clinical and methodological heterogeneity of the eligible studies. Few studies considered similar outcomes, and those that did had either different comparator groups,${ }^{29}{ }^{30}$ differing methods of assessing similar outcomes (eg, survey vs routine data for emergency department (ED) attendance) ${ }^{31} 32$ or concerned complex interventions, the diversity of which would limit the utility of a pooled analysis. ${ }^{31}{ }^{33}$ Consequently, a meta-analysis was deemed inappropriate and we performed a narrative synthesis of the study findings. Studies were grouped by outcome and the strength of the body of evidence for each outcome was assessed using the Grades of Recommendation, Assessment, Development and Evaluation approach. ${ }^{34}$

We constructed a harvest plot post hoc to display the results. Harvest plots use bars representing individual studies placed on a plot matrix to indicate whether the review intervention showed an overall positive, negative or no consistent effect for the outcome in question. They enable data to be summarised when study designs and outcomes are diverse and heterogeneous. ${ }^{35}{ }^{36}$ We used the following criteria to decide how each study should be displayed:

- height of the bar represented the number of participants in the study;

- RCTs were displayed in bold with other designs in grey;

- the ROB for the outcome of each study was indicated as low, moderate or high using a coloured dot above the bar;

- statistically significant differences were displayed as a positive effect if they favoured the intervention; negative if they favoured the comparator and neutral if not statistically significant;

- where some, but not all, findings in a group of outcomes showed a positive or negative effect, bars were hatched to indicate inconsistency.

\section{RESULTS}

\section{Study selection}

The results of abstract and full-text screening are shown in the PRISMA diagram in figure 1. A full list of studies excluded at full-text level, along with reasons for exclusion, is shown in online supplementary file 2 .

\section{Description of studies}

Sixteen papers were eligible for inclusion which described 11 unique studies. ${ }^{29-33}$ 37-47 Ten studies were from the USA ${ }^{29} 30323337-47$ and one from UK. ${ }^{31}$ Eight were RCTs, two quasi-experimental and one was a pilot study.

Three studies included a range of NCDs. ${ }^{31-33}$ None of these studies included specific diagnoses as inclusion criteria, but rather recruited at hospital admission or from homeless accommodation targeting access to community health services. It was not specified if participants included also had LT-CDs. The three studies including a range of NCDs each focused on access to care and services. Identification and management of health needs were included in this, however, the interventions did not target specific conditions or management strategies. With the exception of one small $(n=9)$ pilot study in type two diabetes, all other studies focusing on management of specific conditions concerned LT-CDs: four studies concerned latent $\mathrm{TB}^{29} 30$ 37-41; one concerned hepatitis $\mathrm{C}^{46}$; two studies concerned HIV. $^{43-45} 47$

\section{Study populations}

Details of the study populations are summarised in table 1. Sample sizes ranged from 9 to 520. Median age ranged from 37 to 49 years. In all of the studies, the majority of participants were male (percentage male participants ranged from $67 \%$ to $94 \%$ in the intervention groups). Age and sex distributions were consistent with previous literature on homelessness. ${ }^{1}$ Six studies, all from the USA, reported details of ethnicity. ${ }^{29} 3037414346$ African-American participants were the most prevalent in five of these. Only two studies included any detail 

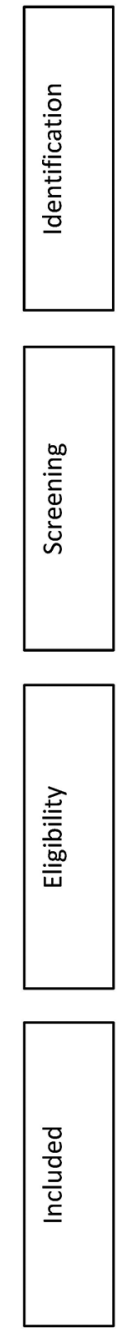

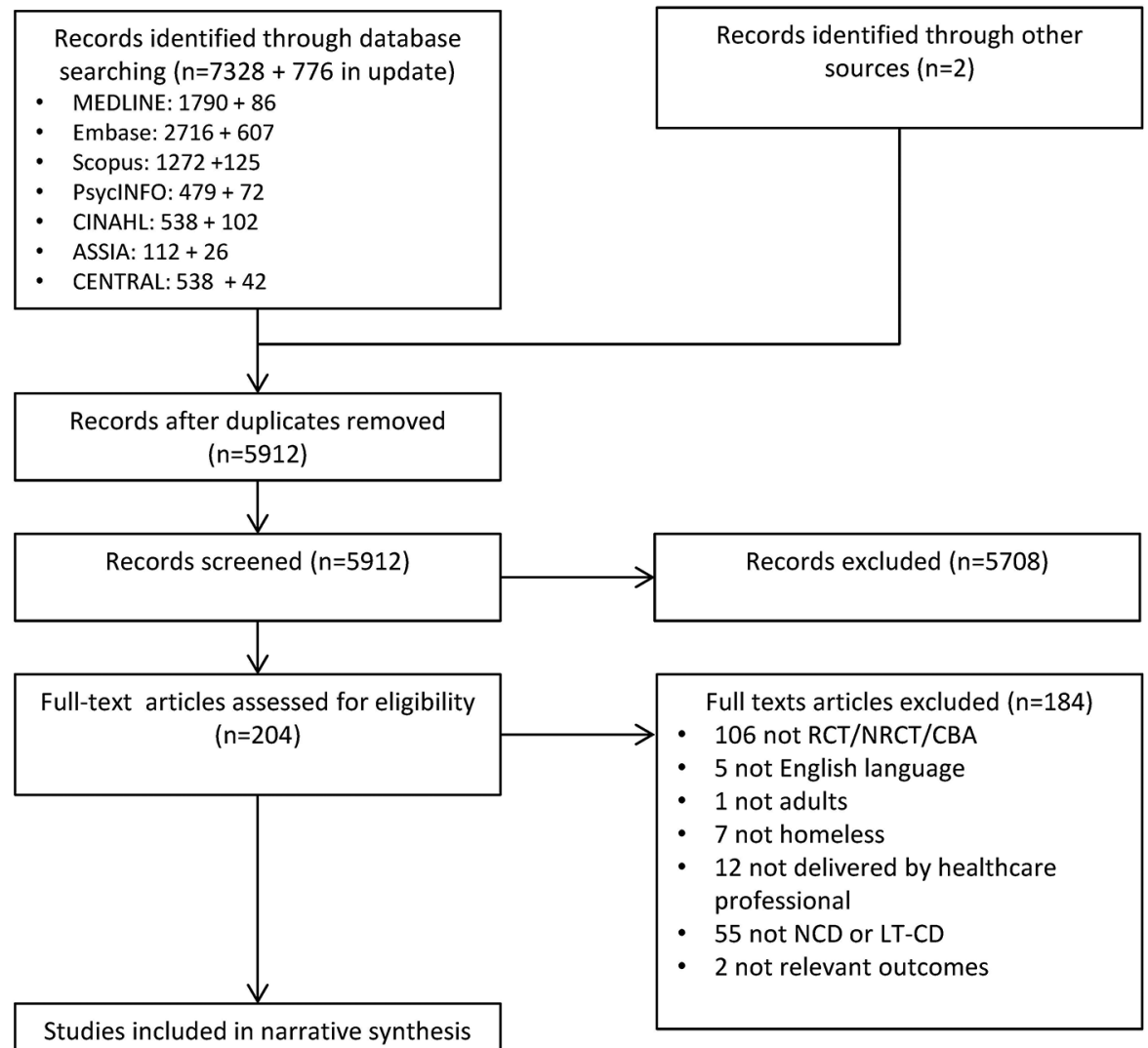

(16 papers reporting 11 studies)

- 8 RCTs

- 2 quasiexperimental studies

- 1 randomised feasibility/pilot study

Figure 1 PRISMA diagram of search results and screening. ASSIA, Applied Social Science Index and Abstracts; CBA, controlled before-after; CENTRAL, Cochrane Central Register of Controlled Trials; CINAHL, Cumulative Index to Nursing and Allied Health Literature; LT-CD, Communicable disease requiring long-term care; NCD, Non-communicable Disease; NRCT, nonrandomised controlled trial; PRISMA, Preferred Reporting Items for Systematic Reviews and Meta-Analyses; RCT, randomised controlled trial.

of comorbidities. ${ }^{31}$ Details of attrition are shown in online supplementary file 3 .

\section{Quality assessment}

Results of the EPOC ROB assessment for each of the included studies are shown in table 2. None of the included studies scored low risk for each of the criteria. These were used to inform outcome-level ROB assessment. These are displayed, along with justification, in online supplementary file 3 .

\section{Intervention components and theoretical underpinnings}

Multidisciplinary teams including both a physician and nurse working alongside social workers delivered two of the interventions. ${ }^{31} 32$ The nine remaining interventions were delivered primarily by a nurse, alone ${ }^{46} 47$ or alongside psychiatrists, ${ }^{43}$ peer-health advisors ${ }^{29} 3041$ or outreach workers. ${ }^{37}$

Each of the studies described interventions that were complex and included multiple components. These included changes to how, and where, care was delivered, the personnel delivering care, how care delivery was coordinated and the provision of financial support. The components of the EPOC taxonomy relating to each of the interventions are shown in table 3, along with a summary of the intervention and control interventions. Descriptions of the specific aspects of each intervention relating to the taxonomy are shown in online supplementary file 4.

Four of the 11 studies reported an explicit theoretical framework underpinning the intervention (table 3). These included the comprehensive health seeking and coping paradigm underpinning two of the studies, and self-efficacy theory and the health belief model each underpinning one intervention.

\section{Impact of interventions on healthcare outcomes}

The overall findings of the included studies for impact on unscheduled healthcare utilisation, adherence or 


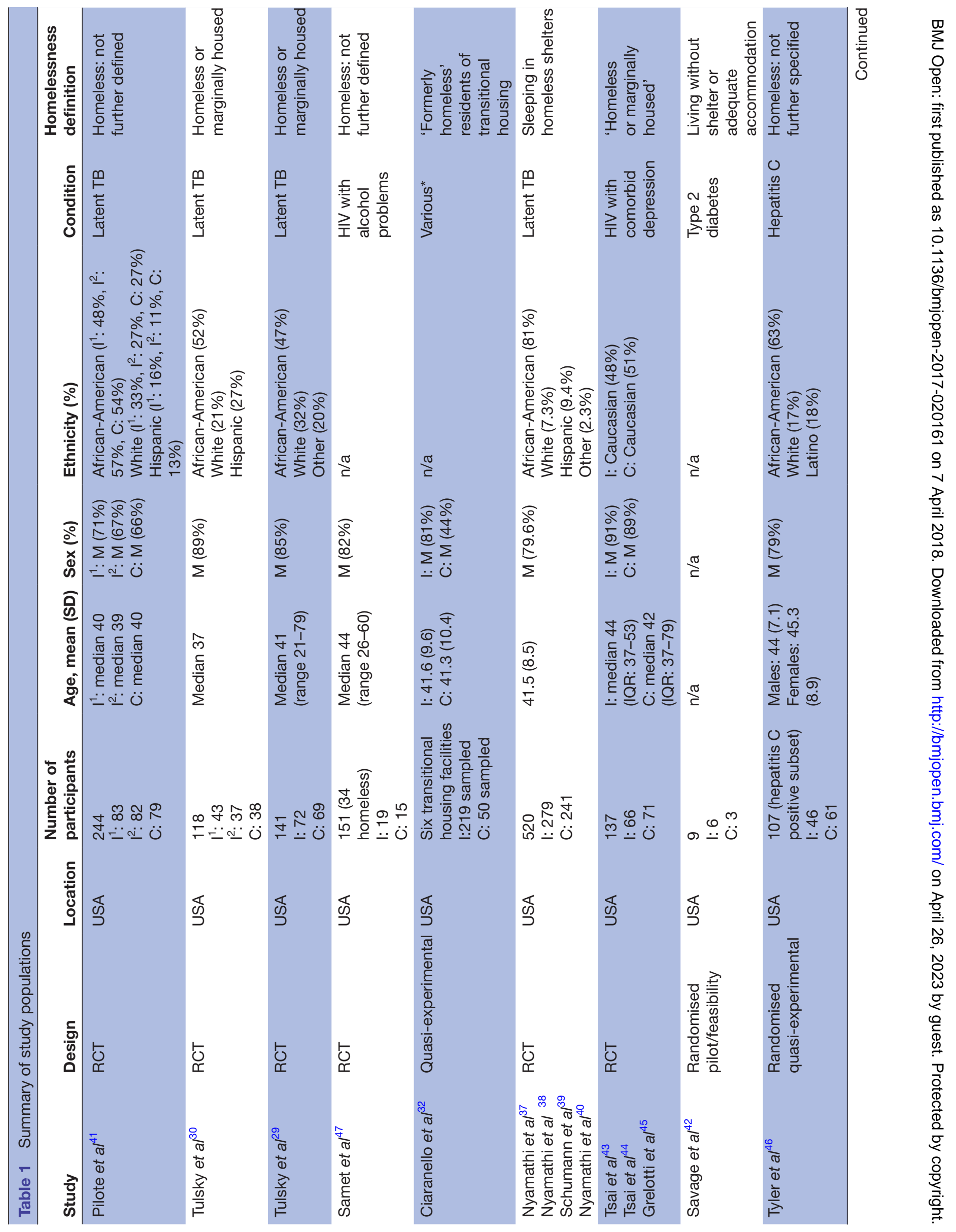




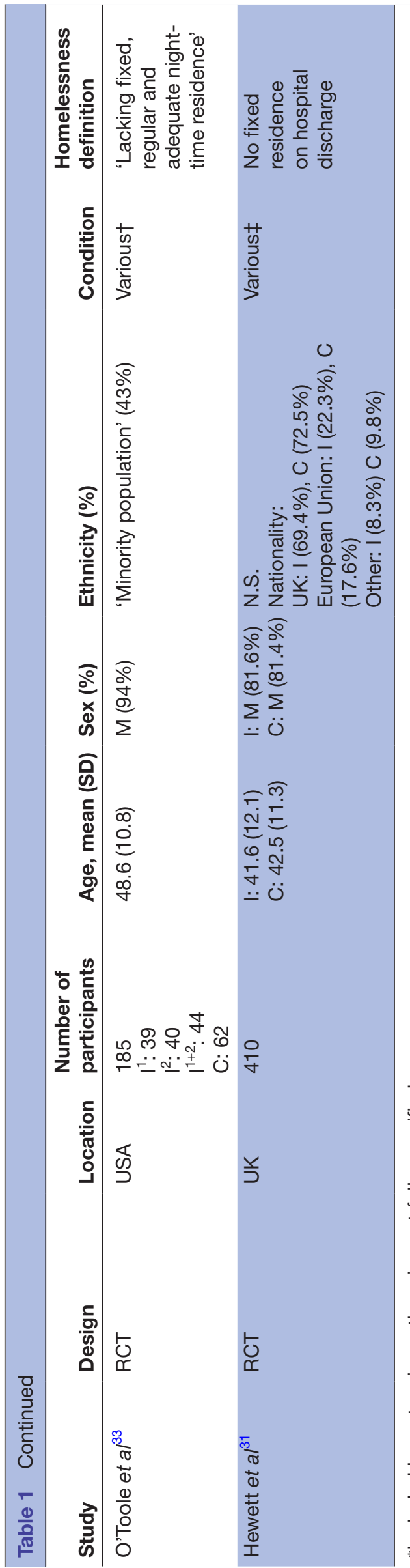

官 outcome.

\section{Primary review outcomes \\ Unscheduled healthcare utilisation}

Three studies assessed the impact of interventions on hospital admissions and ED attendance. ${ }^{31-33}$ None focused on a specific condition, however, participants reported a range of NCDs and each intervention included identification and engagement with medical, as well as wider needs. The highest quality evidence was from two RCTs, neither of which showed any significant reduction in unscheduled healthcare utilisation. ${ }^{31} 33$ One RCT evaluated a multidisciplinary, multicomponent intervention targeting patients in two inner-city hospitals involving goal setting, discharge planning and liaising with community services. ${ }^{31}$ Neither hospital admissions nor ED attendance after 1 year were significantly different compared with usual care. The other RCT was a four-arm trial comparing usual care; a brief nurse-led physical health needs assessment; a guided orientation to clinical facilities with introduction to staff; and clinic orientation in combination with the physical health assessment. ${ }^{33}$ Hospital admissions and ED attendance were assessed at 6 months postintervention in a post hoc analysis and showed no significant difference to usual care. A third study, with a quasi-experimental design and high ROB, concerned a 'comprehensive health assessment' delivered to residents at transitional housing facilities. ED attendances were reportedly lower at 18 months follow-up, but not at 6 months. There was no difference in hospitalisation at either follow-up point.

Taken together the available evidence does not suggest that the multidisciplinary, multifaceted interventions described reduced rates of unscheduled healthcare utilisation. The overall confidence in the estimate of effect is low. There were no studies targeting specific NCD or LT-CDs.

\section{Secondary review outcomes} Access to primary healthcare

One RCT, including a range of NCDs, concerned access to primary healthcare. ${ }^{33} \mathrm{~A}$ brief nurse-led physical health needs assessment, a guided orientation to clinical facilities with introduction to staff and clinic orientation in combination with the physical health assessment were compared with usual care. All three intervention groups showed higher uptake of primary healthcare services after 6 months with clinic orientation alone and in combination with a physical health assessment significantly improving primary care access in adjusted analyses. Overall confidence in effect for improvement in this outcome was high, but limited to one study so should be interpreted with caution. 


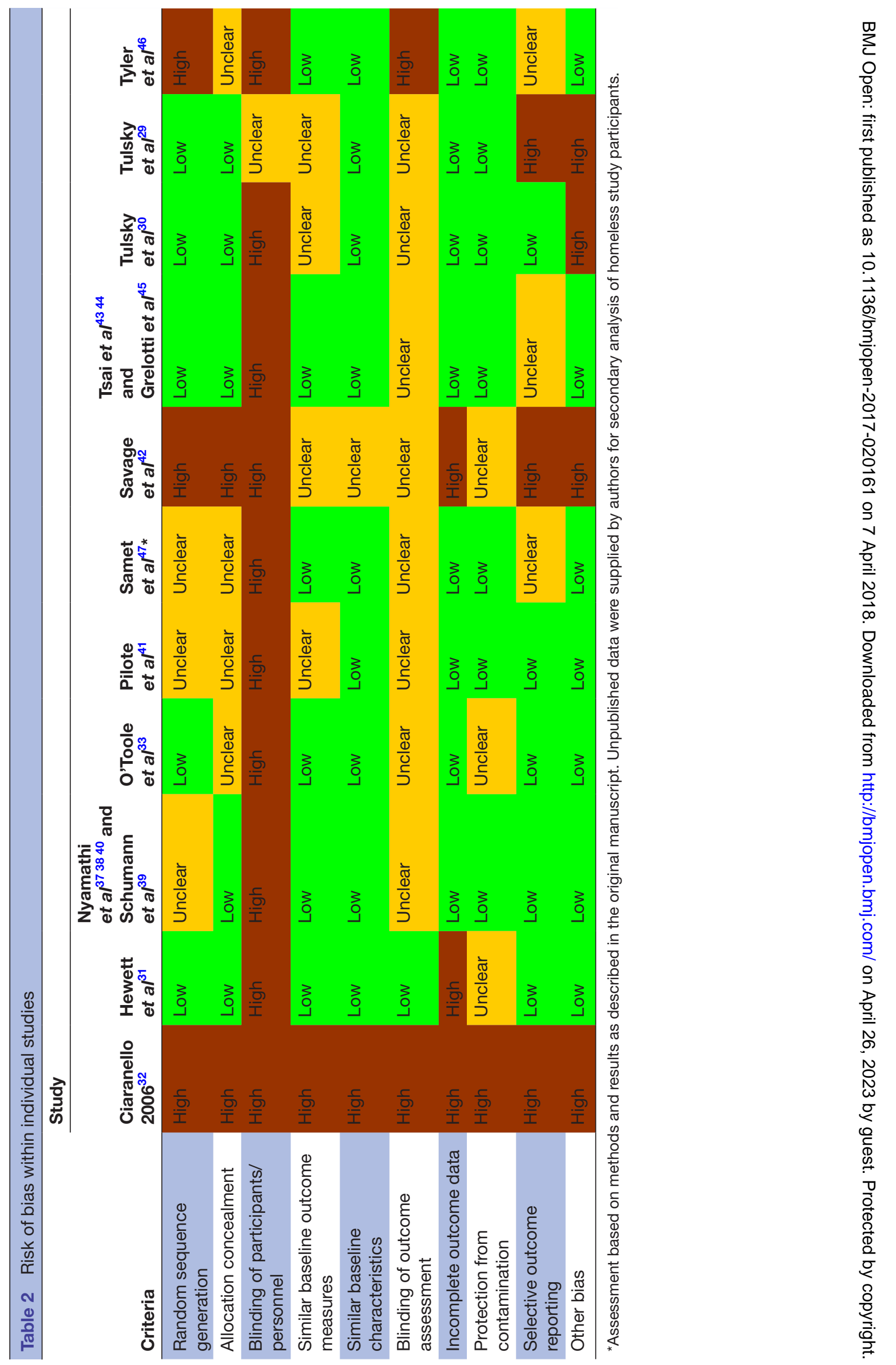




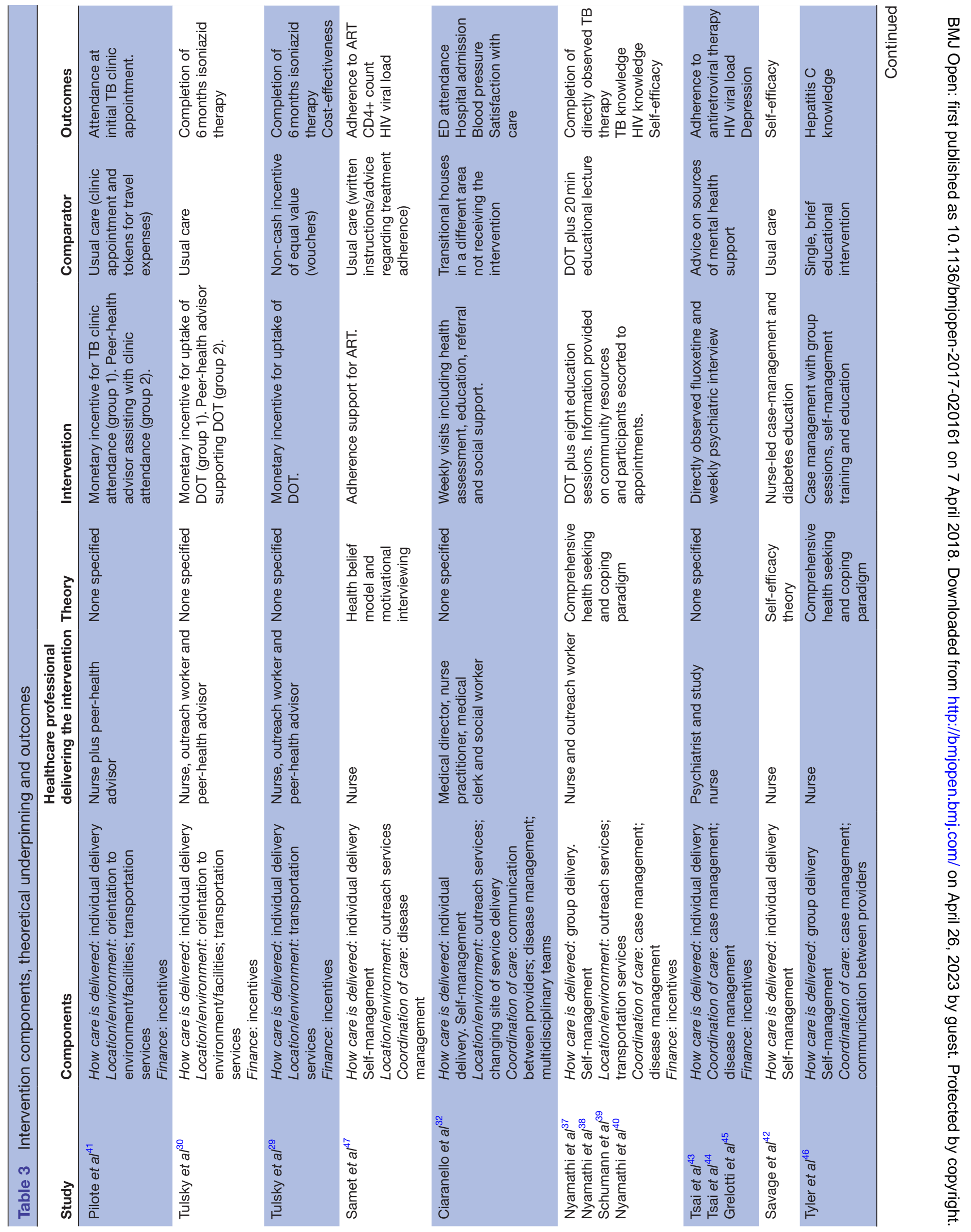




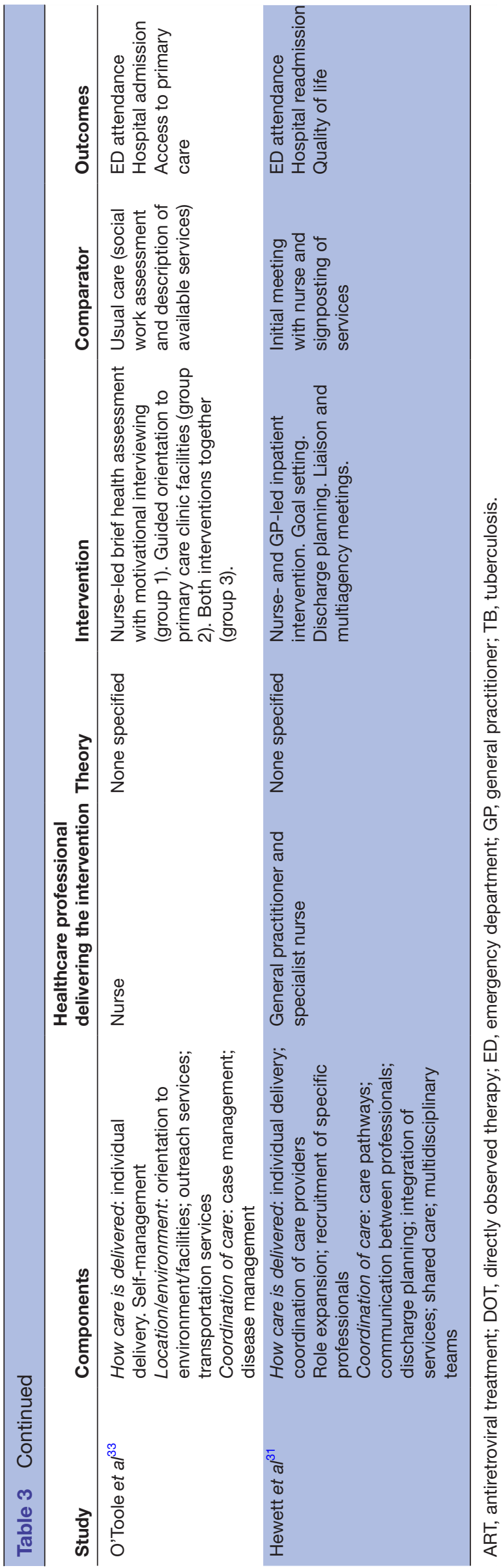

Adherence to specific treatment

Six studies (seven papers), all of which concerned LT-CDs, assessed adherence to treatment or attendance at appointments. ${ }^{29} 303741434447$ Four recruited patients with latent $\mathrm{TB}$ undergoing directly observed therapy (DOT),${ }^{29} 303741$ one included participants with HIV and alcohol problems ${ }^{47}$ and one (two papers) concerned participants with HIV and comorbid depression. ${ }^{43}{ }^{44}$ Of the TB studies, three were conducted by the same research group and assessed the impact of monetary incentives (cash and/or voucher) on attendance at initial TB clinic follow-up ${ }^{41}$ or on completion of DOT with isoniazid. ${ }^{29} 30$ Clinic attendance and DOT completion rates were significantly higher with cash incentives compared with usual care or peer-health advisors. ${ }^{30}$ There was no statistically significant difference in DOT completion between cash and voucher incentives. ${ }^{29}$ Details of the availability to the participants of social security or other sources of financial support are not described in either study. Although the cash incentive and delivery of the intervention were similar in both studies assessing DOT completion, the completion rate in the intervention group differed widely between the two studies (44\% and $89 \%$, respectively).$^{29} 30$ The authors speculate that the location of the clinic (the higher completion rate being in an area more accessible and frequented by people who are homeless) or alterations in the follow-up protocol for non-attendees may explain the differences.

The final study concerning TB evaluated the impact of a nurse-led case management intervention on completion of latent TB treatment and TB knowledge (described in the Knowledge and self-efficacy section). They found odds of DOT completion were three times greater with the intervention compared with usual care. ${ }^{37}$

An RCT concerning people with HIV and comorbid depression assessed fluoxetine prescription and weekly psychiatric evaluation compared with the provision of information about how to access local psychology services without the prescription of fluoxetine. Both arms were given a weekly cash incentive for attending. Outcomes included rate of uptake of antiretroviral treatment (ART), and adherence to ART (assessed by unannounced pill counts) for those receiving treatment. Neither outcome was significantly different between the groups despite an improvement in depression severity and remission in the fluoxetine group. ${ }^{434}$

Finally, an RCT aimed at supporting antiretroviral medication adherence among HIV-positive participants with a history of alcohol dependence or harmful drinking showed no change in antiretroviral adherence ${ }^{47}$ Findings were similar to a secondary analysis of participants who described themselves as homeless (unpublished results).

Overall, there is a moderate level of evidence for interventions improving adherence to treatment for latent TB, including a case-management educational approach and provision of monetary incentives (cash or non-cash). However, the efficacy of such interventions may be dependent on the social and cultural context in which it 
Harvest Plot: Summary of Impact of Interventions Organised by Outcome and Content

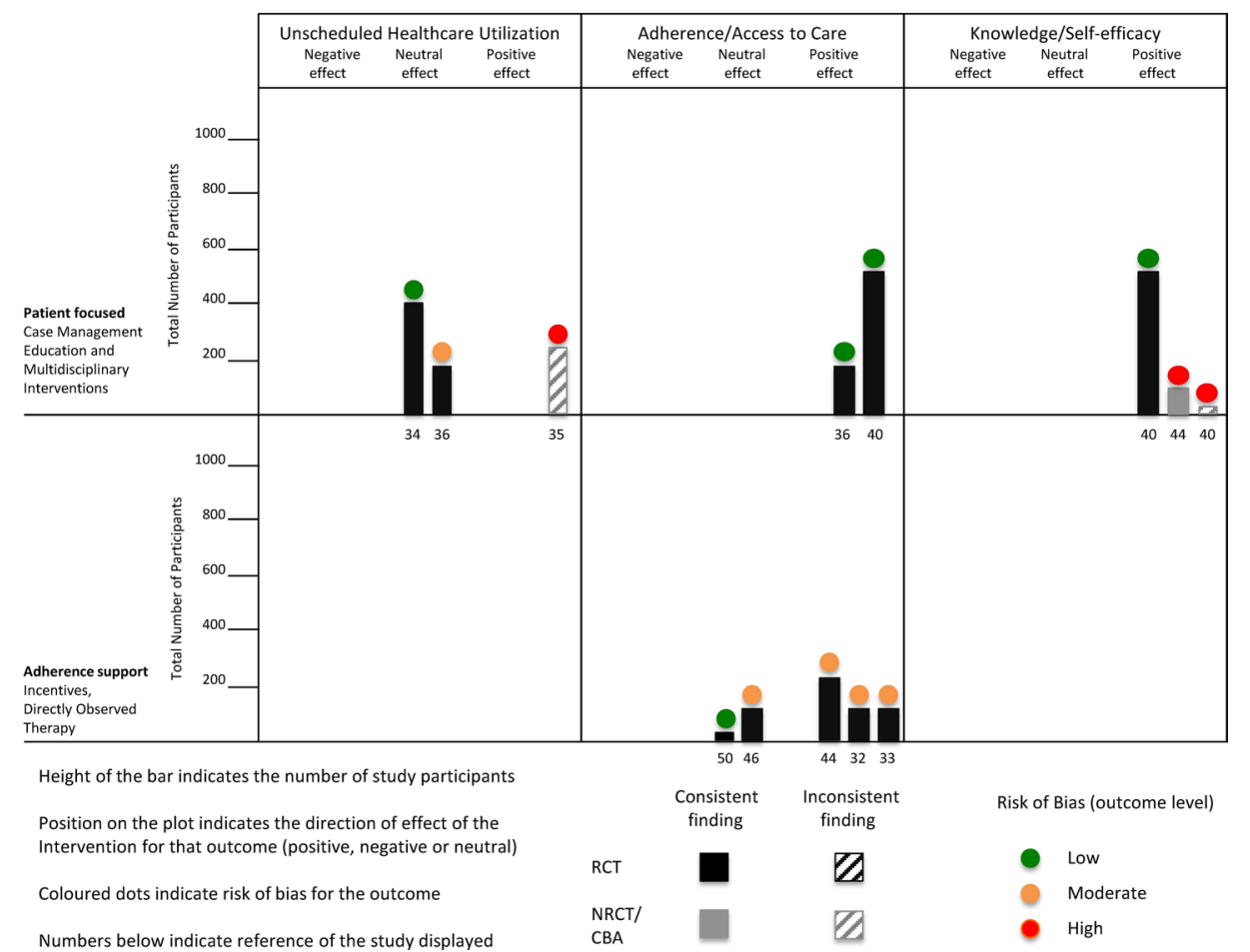

Figure 2 Harvest plot of findings of included studies. CBA, controlled before-after study; NRCT, non-randomised controlled trial; RCT, randomised controlled trial.

is delivered (highlighted by variation in completion rates between evaluations of similar interventions), of which there is limited description in the available studies.

\section{Knowledge and self-efficacy}

Three studies (five papers) assessed the impact of interventions on disease-specific knowledge and self-efficacy. ${ }^{37-39} 4246$ Two (four papers) concerned LT-CDs (TB, HIV and hepatitis) and one concerned type 2 diabetes. Two were trials incorporating nurse-led case management (for patients with latent $\mathrm{TB}$ or hepatitis $\mathrm{C}$, respectively) combined with a regular educational intervention focusing on self-management, self-esteem, communication skills and social support. One was an RCT focusing on DOT for latent TB and assessed the impact on TB knowledge in all participants. ${ }^{37}$ The intervention also involved HIV education and the impact of this was evaluated in a subset judged to be 'at risk' of HIV (ie, sexually active or known to be intravenous drug users). Two analyses using structural equation modelling showed that the nurse-led case management intervention was associated with greater improvement in TB knowledge ${ }^{38}$ and in HIV knowledge in the 'at risk' subset. ${ }^{39}$ The latter also showed improved self-efficacy for condom use. ${ }^{39}$ The other evaluated a similar approach concerning hepatitis education for participants enrolled in a hepatitis $\mathrm{A} / \mathrm{B}$ vaccination programme (only the hepatitis $\mathrm{C}$ positive subset was included in this review). ${ }^{46}$ The case-management group showed a greater improvement in hepatitis C knowledge than the control group. However, the randomisation procedure was designed for the vaccine trial, not for the evaluation of the case-management intervention, and the statistical analysis was not designed to compare the intervention with control in the hepatitis $\mathrm{C}$ subset alone. ${ }^{46}$

The third study reported improved knowledge in a small $(n=9)$ pilot study using a self-efficacy-based approach for type 2 diabetes mellitus. However, the small sample size meant there was insufficient power to detect any difference between groups and there was incomplete reporting of outcomes and no clear comparison is made between the intervention and comparator. ${ }^{42}$

Taken together, there is a moderate quality of evidence showing that an educational case-management approach can improve disease-specific knowledge in the context of specific LT-CDs when delivered alongside wider interventions, such as DOT or a vaccine study. The available studies, however, do not assess the impact on behavioural outcomes or the retention of knowledge beyond the trial period.

\section{Biological markers of disease control}

Two studies (three papers) concerning LT-CDs assessed the impact of interventions on disease control outcomes. One RCT assessed the impact on HIV-1 viral load of directly observed fluoxetine in comorbid HIV and depression. There was no difference in viral suppression between intervention and comparator groups. ${ }^{43-45}$ The other RCT found no difference in viral load or CD4+ count with adherence support for antiretroviral therapy in HIV-infected individuals with a history of alcohol problems. ${ }^{47}$ 


\section{Cost-effectiveness}

Only one study, including participants with a range of conditions including NCDs, assessed cost-effectiveness, within the hospital sector. ${ }^{31}$ Patients in the intervention group also had multiagency care plans devised before, and implemented after hospital discharge. Quality of life was a secondary outcome, with health gain measured by translating generic EQ-5D-5L (five level EuroQol quality of life questionnaire) index scores into generic quality-adjusted life years (QALYs). EQ-5D-5L scores were completed by approximately one quarter of participants in both arms. There was a non-statistically significant increase in EQ-5D-5L scores at follow-up, and there was no impact of the intervention on inpatient costs, therefore the authors compared the costs of the intervention with the effect on health gain as measured by QALYs. On this basis the incremental cost-effectiveness ratio was $£ 26000$ with the authors describing circumstances in which the intervention may be cost-effective, and an accompanying sensitivity analysis. ${ }^{31}$

\section{DISCUSSION}

\section{Summary of findings}

The available evidence from controlled trials of interventions by healthcare professionals improving access to care for people with NCDs who are homeless does not show any convincing effects on unscheduled healthcare utilisation. ${ }^{31-33}$ There is also a lack of evidence to inform the management of specific NCDs in this context. One multidisciplinary intervention did demonstrate improved access to primary healthcare.

Seven interventions were identified targeting specific LT-CDs. All of these involved a nurse primarily delivering the intervention, sometimes with support of peer-health advisors. Patient-centred interventions-incorporating case management, education, self-management support and social support-may improve disease-specific knowledge in TB, HIV and hepatitis C; improve completion of DOT in latent TB and increase access to primary care in combination with clinic orientation. ${ }^{33}$ 37-39 46 Cash and non-cash incentives, in the context of DOT for latent TB, may improve clinic attendance and treatment adherence; however, treatment completion rates vary between different studies of similar interventions. ${ }^{29} 3041$ It is not clear if improvement in these intermediate outcomes impacts other clinical outcomes, or if effects are sustained beyond the course of treatment evaluated in these studies. The impact on mortality was not assessed, and evidence for the impact on biological markers of disease control is limited to a few studies on HIV, which did not show any evidence of benefit on viral load. ${ }^{43} 44$ There was only one study of cost-effectiveness.

\section{Strengths and limitations}

The strengths of this review include a priori methods with a robust process for study identification, appraisal, data extraction and description. ${ }^{25}$ The comprehensive search strategy included database searches supplemented by hand searching, forward citation searching, grey literature and contact with study authors. All screening and data extraction was performed by two reviewers independently. We also described the components of each intervention using a previously defined taxonomy, ${ }^{28}$ which is important when reviewing complex interventions such as those included. ${ }^{48}$ However, many of the findings, particularly those concerning adherence to treatment, were in the context of specific conditions (eg, latent $\mathrm{TB}$ ), included a time-limited course of treatment and were conducted in a single centre. All but one of the included studies were from the USA. As such the findings may not be directly applicable to other disease areas or other health and social care contexts. Limitations in the existing evidence base also meant that we were unable to undertake a formal meta-analysis. Contacting study authors to obtain results pertaining to participants who were homeless (when not reported separately) contributed to the comprehensiveness of the review, however, this strength needs to be balanced against the potential bias of performing post hoc secondary analyses on existing trial data. Furthermore, in such circumstances studies are not specifically powered to assess outcomes in this subgroup.

This review is timely given the increasing number and complexity of health problems among people who are homeless, ${ }^{1}$ the pressure on healthcare services to address this burden and the potentially expanding roles of various healthcare professionals to support management of NCDs and LT-CDs. ${ }^{20}$ However, by focusing on interventions by healthcare professionals this review may overlook evidence for housing or social interventions that may impact on such conditions. ${ }^{5051}$

\section{Implications for practice, policy and research}

Despite the social complexity and exclusion that typify the experience of homelessness, a patient-focused case-management approach was shown to positively impact disease-specific knowledge and self-efficacy in the management of selected LT-CDs. ${ }^{37-39} 46$ These interventions were primarily delivered by a study nurse, with or without peer-health advisors, adopting a case-management approach.

It is not clear to what extent the findings presented here are generalisable to wider social or healthcare contexts, or to other conditions. The evidence for improved adherence was predominantly in the context of DOT for latent $\mathrm{TB}$ and in some cases involved cash incentives. Further research would be required to establish whether these principles of adherence support are transferable to the management of NCDs. Furthermore, the potential efficacy of cash incentives will vary between societal contexts where access to, and the extent of, financial support varies widely. Finally, the available literature focuses mainly on the role of nurses and physicians, often alongside other ancillary staff (such as peer advisors, case managers and care coordinators), with little consideration of the 
potential role of other healthcare professionals, for example, pharmacists.

The extent to which the improvements in knowledge or adherence that have been demonstrated may impact on physical or behavioural outcomes has not been evaluated. This raises the question of how such issues may be best addressed by future research. It is likely, given their apparent scarcity, that further evaluation of complex interventions to address both NCD and LT-CDs management (including aspects of randomisation, longer follow-up and consideration of broader outcomes) will be needed to inform practice. Based on existing patterns of need and service utilisation, as well as the need to demonstrate effectiveness and cost-effectiveness of novel models of care, well-designed and conducted studies following a framework for testing complex interventions ${ }^{49}$ for people who are homeless are overdue.

However, the intrinsic complexity of the experience of homelessness, and the impact this has on health, may require a broader methodological approach (eg, realist synthesis) to understand the context and process of potential interventions in this area.

\section{CONCLUSIONS}

Trials of interventions delivered by healthcare professionals targeting NCD in people who are homeless do not show convincing evidence of the primary outcome measure for this review-an impact on unscheduled healthcare utilisation. Despite their high prevalence and associated morbidity and mortality, little evidence was identified to inform the management of specific NCDs.

In the context of specific LT-CDs (HIV, TB and hepatitis $\mathrm{C}$ ), patient-centred case-management interventions may improve knowledge and self-efficacy. Available evidence supports interventions delivered by a nurse and incorporating peer-health advisors. These interventions, as well as incentives, may also improve adherence in specific contexts. The impact on biological outcomes and mortality remains largely unexplored, as does the effectiveness of alternative models of care involving different professions. The economic impact of successful interventions is also largely unexplored. Future complex intervention evaluation research is needed to test innovative models of care, and expand those interventions showing promise, into diverse health and social care contexts.

Acknowledgements We would like to acknowledge Catriona Deenoon, librarian for NHS Greater Glasgow and Clyde, for her support and advice in carrying out the scoping searches, designing the search strategy and piloting and finalising the search terms.

Contributors All authors listed fulfil the ICMJE criteria for authorship. RL conceived the initial idea. All authors contributed to the conception and design of the proposed study. PH, LY, RE, AEW, FSM and RL contributed to the development of data sources and search strategy. PH, LY, RE, AEW, FSM and RL developed and refined the inclusion criteria. PH, LY, RE, LG, FSM and RL developed the data extraction template which was piloted by $P H, L Y$ and LG. PH, LY, RE and RL screened titles, abstract and full texts. PH, LY and LG completed data extraction and quality assessment on all included studies. PH wrote the first draft of the manuscript. All authors critically reviewed this and subsequent drafts of the manuscript and provided input into its content. All authors approved the final version of the manuscript to be published. RL is the guarantor of the review. All authors accept accountability for the accuracy of the findings presented.

Funding This research received no specific grant from any funding agency in the public, commercial or not-for-profit sectors.

Competing interests None declared.

Patient consent Not required.

Provenance and peer review Not commissioned; externally peer reviewed.

Data sharing statement Full details of the screening process are detailed in the supplementary appendices. Any additional detail will be available on request from the corresponding author.

Open Access This is an Open Access article distributed in accordance with the Creative Commons Attribution Non Commercial (CC BY-NC 4.0) license, which permits others to distribute, remix, adapt, build upon this work non-commercially, and license their derivative works on different terms, provided the original work is properly cited and the use is non-commercial. See: http://creativecommons.org/ licenses/by-nc/4.0/

(c) Article author(s) (or their employer(s) unless otherwise stated in the text of the article) 2018. All rights reserved. No commercial use is permitted unless otherwise expressly granted.

\section{REFERENCES}

1. Fazel S, Geddes JR, Kushel M. The health of homeless people in high-income countries: descriptive epidemiology, health consequences, and clinical and policy recommendations. Lancet 2014;384:1529-40.

2. Nusselder WJ, Slockers MT, Krol L, et al. Mortality and life expectancy in homeless men and women in Rotterdam: 2001-2010. PLoS One 2013;8:e73979.

3. Nielsen SF, Hjorthøj CR, Erlangsen A, et al. Psychiatric disorders and mortality among people in homeless shelters in Denmark: a nationwide register-based cohort study. Lancet 2011;377:2205-14.

4. Lebrun-Harris LA, Baggett TP, Jenkins DM, et al. Health status and health care experiences among homeless patients in federally supported health centers: findings from the 2009 patient survey. Health Serv Res 2013;48:992-1017.

5. Barnett K, Mercer SW, Norbury M, et al. Epidemiology of multimorbidity and implications for health care, research, and medical education: a cross-sectional study. Lancet 2012;380:37-43.

6. Dixon L, Postrado L, Delahanty J, et al. The association of medical comorbidity in schizophrenia with poor physical and mental health. $J$ Nerv Ment Dis 1999;187:496-502.

7. Bellis MA, Hughes K, Leckenby N, et al. Measuring mortality and the burden of adult disease associated with adverse childhood experiences in England: a national survey. J Public Health 2015;37:445-54.

8. Nyamathi AM, Dixon EL, Robbins W, et al. Risk factors for hepatitis $\mathrm{C}$ virus infection among homeless adults. J Gen Intern Med 2002;17:134-43.

9. Luchenski S, Maguire N, Aldridge RW, et al. What works in inclusion health: overview of effective interventions for marginalised and excluded populations. Lancet 2017:266-80.

10. Lee TC, Hanlon JG, Ben-David J, et al. Risk factors for cardiovascular disease in homeless adults. Circulation 2005;111:2629-35.

11. Kim DH, Daskalakis C, Plumb JD, et al. Modifiable cardiovascular risk factors among individuals in low socioeconomic communities and homeless shelters. Fam Community Health 2008;31:269-80.

12. Argintaru N, Chambers C, Gogosis E, et al. A cross-sectional observational study of unmet health needs among homeless and vulnerably housed adults in three Canadian cities. BMC Public Health 2013;13:577

13. Kushel MB, Vittinghoff E, Haas JS. Factors associated with the health care utilization of homeless persons. JAMA 2001;285:200-6.

14. Gelberg L, Andersen RM, Leake BD. The Behavioral model for vulnerable populations: application to medical care use and outcomes for homeless people. Health Serv Res 2000;34:1273-302.

15. Brett T, Arnold-Reed DE, Troeung L, et al. Multimorbidity in a marginalised, street-health Australian population: a retrospective cohort study. BMJ Open 2014;4:e005461.

16. Wright NM, Tompkins CN. How can health services effectively meet the health needs of homeless people? $\mathrm{Br} J$ Gen Pract 2006;56:286-93. 
17. Hwang SW, Burns T. Health interventions for people who are homeless. The Lancet 2014;384:1541-7.

18. Hewett NC. How to provide for the primary health care needs of homeless people: what do homeless people in Leicester think? Br J Gen Pract 1999;49:819.

19. Hewett N, Halligan A, Boyce T. A general practitioner and nurse led approach to improving hospital care for homeless people. BMJ 2012;345:e5999.

20. Courtenay M, Carey N, Stenner K. An overiew of non medical prescribing across one strategic health authority: a questionnaire survey. BMC Health Serv Res 2012;12:138.

21. Queen AB, Lowrie R, Richardson J, et al. Multimorbidity, disadvantage, and patient engagement within a specialist homeless health service in the UK: an in-depth study of general practice data. BJGP Open 2017:BJGP-2016-0641.

22. Hwang SW, Wilkins R, Tjepkema M, et al. Mortality among residents of shelters, rooming houses, and hotels in Canada: 11 year follow-up study. BMJ 2009;339:b4036.

23. Hwang SW, Tolomiczenko G, Kouyoumdjian FG, et al. Interventions to improve the health of the homeless: a systematic review. $A m \mathrm{~J}$ Prev Med 2005;29:311-75.

24. Fitzpatrick-Lewis D, Ganann R, Krishnaratne S, et al. Effectiveness of interventions to improve the health and housing status of homeless people: a rapid systematic review. BMC Public Health 2011;11:638.

25. Hanlon P, Yeoman L, Esiovwa R, et al. Interventions by healthcare professionals to improve management of physical long-term conditions in adults who are homeless: a systematic review protocol. BMJ Open 2017;7:e016756.

26. Moher D, Liberati A, Tetzlaff J, et al. Preferred reporting items for systematic reviews and meta-analyses: the PRISMA statement. Ann Intern Med 2009;151:264-9.

27. Lombe M, Nebbitt VE, Sinha A, et al. Examining effects of food insecurity and food choices on health outcomes in households in poverty. Soc Work Health Care 2016;55:440-60.

28. Effective Practice and Organisation of Care (EPOC). EPOC resources for review authors. Oslo: Norwegian Knowledge Centre for the Health Services, 2015. http://epoc.cochrane.org/epoc-specific-resourcesreview-authors (accessed Dec 2017).

29. Tulsky JP, Hahn JA, Long HL, et al. Can the poor adhere? Incentives for adherence to TB prevention in homeless adults. Int $J$ Tuberc Lung Dis 2004;8:83-91.

30. Tulsky JP, Pilote L, Hahn JA, et al. Adherence to isoniazid prophylaxis in the homeless: a randomized controlled trial. Arch Intern Med 2000;160:697-702.

31. Hewett N, Buchman P, Musariri J, et al. Randomised controlled trial of GP-led in-hospital management of homeless people ('Pathway'). Clin Med 2016;16:223-9.

32. Ciaranello AL, Molitor F, Leamon M, et al. Providing health care services to the formerly homeless: a quasi-experimental evaluation. $J$ Health Care Poor Underserved 2006;17:441-61.

33. O'Toole TP, Johnson EE, Borgia ML, et al. Tailoring outreach efforts to increase primary care use among homeless veterans: results of a randomized controlled trial. J Gen Intern Med 2015;30:886-98.

34. Guyatt GH, Oxman AD, Vist GE, et al. GRADE: an emerging consensus on rating quality of evidence and strength of recommendations. BMJ 2008;336:924-6.
35. Crowther M, Avenell A, MacLennan G, et al. A further use for the Harvest plot: a novel method for the presentation of data synthesis. Res Synth Methods 2011;2:79-83.

36. Ogilvie D, Fayter D, Petticrew M, et al. The harvest plot: a method for synthesising evidence about the differential effects of interventions. BMC Med Res Methodol 2008;8:8.

37. Nyamathi AM, Christiani A, Nahid P, et al. A randomized controlled trial of two treatment programs for homeless adults with latent tuberculosis infection. Int J Tuberc Lung Dis 2006;10:775-82.

38. Nyamathi A, Stein JA, Schumann A, et al. Latent variable assessment of outcomes in a nurse-managed intervention to increase latent tuberculosis treatment completion in homeless adults. Health Psychol 2007;26:68-76.

39. Schumann A, Nyamathi A, Stein JA. HIV risk reduction in a nurse case-managed TB and HIV intervention among homeless adults. $J$ Health Psychol 2007;12:833-43.

40. Nyamathi A, Nahid P, Berg J, et al. Efficacy of nurse case-managed intervention for latent tuberculosis among homeless subsamples. Nurs Res 2008;57:33-9.

41. Pilote L, Tulsky JP, Zolopa AR, et al. Tuberculosis prophylaxis in the homeless. A trial to improve adherence to referral. Arch Intern Med 1996;156:161-5.

42. Savage $C, X u Y$, Richmond MM, et al. A pilot study: retention of adults experiencing homelessness and feasibility of a CDSM diabetes program. $J$ Community Health Nurs 2014;31:238-48.

43. Tsai AC, Karasic DH, Hammer GP, et al. Directly observed antidepressant medication treatment and HIV outcomes among homeless and marginally housed HIV-positive adults: a randomized controlled trial. Am J Public Health 2013;103:308-15.

44. Tsai AC, Mimiaga MJ, Dilley JW, et al. Does effective depression treatment alone reduce secondary HIV transmission risk? Equivocal findings from a randomized controlled trial. AIDS Behav 2013;17:2765-72.

45. Grelotti DJ, Hammer GP, Dilley JW, et al. Does substance use compromise depression treatment in persons with HIV? Findings from a randomized controlled trial. AIDS Care 2017;29:273-9.

46. Tyler D, Nyamathi A, Stein JA, et al. Increasing hepatitis $C$ knowledge among homeless adults: results of a communitybased, interdisciplinary intervention. J Behav Health Serv Res 2014:41:37-49.

47. Samet JH, Horton NJ, Meli S, et al. A randomized controlled trial to enhance antiretroviral therapy adherence in patients with a history of alcohol problems. Antivir Ther 2005;10:83-93.

48. Shepperd S, Lewin S, Straus S, et al. Can we systematically review studies that evaluate complex interventions? PLoS Med 2009;6:e1000086.

49. Hoffmann TC, Glasziou PP, Boutron I, et al. Better reporting of interventions: template for intervention description and replication (TIDieR) checklist and guide. BMJ 2014;348:91687.

50. Kushel MB, Colfax G, Ragland K, et al. Case management is associated with improved antiretroviral adherence and CD4+ cell counts in homeless and marginally housed individuals with HIV infection. Clin Infect Dis 2006;43:234-42.

51. Wolitski RJ, Kidder DP, Pals SL, et al. Randomized trial of the effects of housing assistance on the health and risk behaviors of homeless and unstably housed people living with HIV. AIDS Behav 2010;14:493-503. 